\title{
CONTINUOUS GROUPS.
}

SopHus Lre: Vorlesungen über continuirliche Gruppen mit geometrischen und anderen Anwendungen. Bearbeitet und ausgegeben von Dr. Georg Schefrzers. Leipzig, Teubner, 1893. 8vo, pp. $x i+810$.

IN the theory of transformation-groups we owe to Professor Lie a most important and interesting department of modern mathematics. The importance of the group idea itself has long been recognized in its application to the theory of substitutions, and some continuous transformations, such as the pedal transformation, were in use before Lie's work, but were used without their connection with the group idea being discovered, and this discovery and the presentation of the results of it in a systematic form are due to Professor Lie.

As far as the finite transformation-groups are concerned, these results have been gathered from the various journals in which they first appeared, and are available in a complete analytical form in the "Theory of Transformation Groups." *

In this book Professor Engel has accomplished with remarkable success the difficult task of presenting the general theory in a rigorous and clear manner. This general treatment, however, involves no small amount of difficulty, and to furnish an introduction to it the "Lectures on Continuous Groups" was written. At the same time it was aimed in this book alone to give, in outline at least, the general theory and indicate some lines in which it may be applied. Both of these ends Dr. Scheffers has successfully accomplished. The first part takes the reader through a discussion of transformation-groups in one and two variables, devoting especial attention to the projective groups and their most important subgroups. All the necessary principles of the projective geometry are derived as used, and a very elementary knowledge of mathematics is sufficient to enable the student to read this portion of the book. In the second part the general theory of continuous groups in $n$ variables is dealt with. It is shown that if

$$
x_{i}^{\prime}=f_{i}\left(x_{1} \ldots x_{n} a_{1} \ldots a_{r}\right), i=1.2 \ldots n
$$

are the equations defining a continuous group, that there exist $r . n$ relations

$$
\frac{\partial x_{i}^{\prime}}{\partial a_{k}}=\sum_{1}^{r} \psi_{j k}\left(a_{1} \ldots a_{r}\right) \xi_{j i}\left(x_{1} \ldots x_{n}\right)\left\{\begin{array}{l}
i=1.2 \ldots n \\
k=1.2 \ldots r
\end{array}\right.
$$

\footnotetext{
* Reviewed by Dr. Chapman in the Bulletin of the Nero York Mathematical Society, vol. 2, No. 4.
} 
such that no relations of the form

hold.

$$
e_{1} \xi_{1 i}\left(x^{\prime}\right)+\ldots+e_{r} \xi_{r i}\left(x^{\prime}\right) \equiv 0 \quad(i=1.2 \ldots n)
$$

Secondly, that if $X_{1} f \ldots X_{r} f$ represent the infinitesimal transformations of the group, relations of the form

$$
\begin{gathered}
\left(X_{i} X_{k}\right) f=X_{i}\left(X_{k} f\right)-X_{k}\left(X_{i} f\right)=\sum_{1}^{r} s c_{i k s} X_{s} f \\
(i, k=1.2 \ldots r)
\end{gathered}
$$

always hold where the $c_{i k s}$ are constant; and finally, that among these constants we have the relations

and

$$
c_{i k l}+c_{k i l}=0
$$

$$
\begin{gathered}
\sum_{1}^{r} s\left(c_{i k s} c_{s l t}+c_{k l s} c_{s i t}+c_{l i s} c_{s k t}\right)=0 \\
(i, k, l, t=1.2 \ldots r) .
\end{gathered}
$$

These three theorems are known as the fundamental theorems of the theory of continuous groups.

Ordinary and differential invariants of a group are defined, the determination of subgroups discussed, and applications to the theory of number systems, geometry, and differential equations fill the closing sections. To attempt to give an account of all the subjects treated would be foolish. It will be more interesting to confine our attention to one or two points of fundamental importance, which may be clearly stated in a short space.

A very noticeable characteristic of the book is the free use of geometrical ideas both in illustration and proof. It conforms in this respect very closely to Lie's habits of thinking and lecturing. It is only necessary to call attention to his articles on Line and Sphere Complexes and Minimal Surfaces to show that Lie occupies a leading position among living geometers. His later work, and "The Theory of Transformation Groups," is of such an extreme analytical character that it would be perhaps easier to regard him as essentially an analyst. Such is not the case, however, as geometrical considerations underlie almost all his analytical work, and led him in the first place to the study of continuous groups. An interesting feature of his lecture courses in the University of Leipzig is this preliminary geometrical derivation of the theorem in question, which is followed by its analytical demonstration.

The distinctive characteristic of the continuous groups is 
the infinitesimal transformation, and to the presence of this transformation and the symbol of it which Lie obtains is due the extreme simplicity which most complicated operations assume and the ease of carrying them out. It will be of interest to sketch the derivation of this transformation for the case of two variables as it is carried out in the second section of the "Continuous Groups." Such a group will be defined by two equations of the form

$$
\left\{\begin{array}{l}
x_{1}=\phi\left(x y a_{1} \ldots \ldots a_{r}\right) \\
y_{1}=\psi\left(x y a_{1} \ldots a_{r}\right)
\end{array}\right.
$$

with the $r$ arbitrary constants or parameters $a_{1}, a_{2} \ldots a_{r}$

As these parameters $a_{1} \ldots a_{r}$ may take all values from $-\infty$ to $+\infty$, there will be in all $\infty^{r}$ transformations in the group (1), which is called then an $r$-parameter or $r$-branch group. The group property, namely, that the succession of any two transformations of the form (1) is equivalent to another transformation of the same form, must of course be satisfied by the transformations. If we regard $(x y)$ as the co-ordinates of a point in a plane, the transformation 1) represents geometrically a transformation of the points of the plane. The group property then will be, that if a point $(x y)$ is transformed into $\left(x_{1} y_{1}\right)$, and this in turn into $\left(x_{2} y_{2}\right)$ by two transformations of the group, a third exists which will move $(x y)$ directly into $\left(x_{2} y_{2}\right)$. It will be possible also to carry the point $\left(x_{1} y_{1}\right)$ back into the position $(x y)$, so that in the group to any given transformation its inverse exists, or they arrange themselves in pairs of inverses, and also the identical transformation which leaves $(x y)$ unchanged. As all these movements are continuous, a point can be moved into a position infinitesimally near. The functions $\phi$ and $\psi$ must be analytical functions of the parameters $a_{1} \ldots a_{r}$, so that an infinitesimal change of these causes only a corresponding infinitesimal change of $\phi$ and $\psi$. For some values of the parameters $a_{1}{ }^{0} \ldots a_{r}{ }^{0}$ we must also have

$$
\left\{\begin{array}{l}
x_{1}=\phi\left(x y a_{1}^{0} \ldots a_{r_{0}^{0}}\right)=\phi\left(x y a^{0}\right)=x \\
y_{1}=\psi\left(x y a_{1}^{0} \ldots a_{r}\right)=\psi\left(x y a^{0}\right)=y
\end{array}\right.
$$

If $\delta a_{k}(k=1.2 \ldots r)$ are quantities which do not vanish identically, but approach zero as a limit, the parameters $a_{k}{ }^{\circ}+\delta a_{k}$ will cause only an infinitesimal change of $x$ and $y$. This change will be represented by

$$
\begin{aligned}
& x_{1}=\phi\left(x y, a^{0}+\delta a\right) \\
& y_{1}=\psi\left(x y, a^{0}+\delta a\right)
\end{aligned}
$$


which may be expanded by Taylor's theorem into a series

whence

$$
\left\{\begin{array}{l}
x_{1}=x+\sum_{1}^{r} i \frac{\partial \phi\left(x y a^{0}\right)}{\partial a_{i}} \delta a_{i}+\ldots \\
y_{1}=y+\sum_{1}^{r} \frac{\partial \psi\left(x y a^{0}\right)}{\delta a_{i}} \delta a_{i}+\ldots
\end{array}\right.
$$

or

$$
\left\{\begin{array}{l}
\delta x=x_{1}-x=\sum_{1}^{r} \frac{\partial \phi\left(x y a^{0}\right)}{\delta a_{i}} \delta a_{i}+\ldots \\
\delta y=y_{1}-y=\sum_{1}^{r} \frac{\partial \psi\left(x y a^{0}\right)}{\delta a_{i}} \delta a_{i}+\ldots
\end{array}\right.
$$

$$
\left\{\begin{aligned}
\delta x & =\sum_{1}^{r} i \xi_{i}(x y) \delta a_{i}+\ldots \\
\delta y & =\sum_{1}^{r} i \eta_{i}(x y) \delta a_{i}+\ldots
\end{aligned}\right.
$$

Replacing $\delta a_{i}$ by $\delta a_{i}=e_{i} \delta t+\ldots$, where $\delta t$ fulfils the same conditions as $\delta a_{i}$, we have

$$
\begin{aligned}
\delta x & =\sum_{1}^{r} i e_{i} \xi_{i}(x y) \delta t+\ldots \\
\delta y & =\sum_{1}^{r} i e_{i} \eta_{i}(x y) \delta t+\ldots
\end{aligned}
$$

As $x$ and $y$ receive these increments by the transformation, an arbitrary function of them, $f(x y)$, will receive an increment

where

$$
\begin{aligned}
\delta f & =\frac{\partial f}{\partial x} \delta x+\frac{\partial f}{\partial y} \delta y \\
& =\left(\sum_{1}^{r} i e_{i} \xi_{i}(x y) \frac{\partial f}{\partial x}+\sum_{1}^{r} e_{i} \eta_{i}(x y) \frac{\partial f}{\partial y}\right) \delta t+\ldots \\
& =X f \delta t+\ldots
\end{aligned}
$$

$$
\begin{aligned}
X f & =\sum_{1}^{r} i e_{i}\left(\xi_{i}(x y) \frac{\partial f}{\partial x}+\eta_{i}(x y) \frac{\partial f}{\partial y}\right)=\xi(x y) p+\eta(x y) q \\
p & =\frac{\partial f}{\partial x} \quad q=\frac{\partial f}{\partial y} .
\end{aligned}
$$

The expression $X f$ is the increment which any function of $x$ and $y$ receives by the infinitesimal transformation of the 
group (1), divided by a constant $\delta t$, neglecting now terms of orders higher than the first in $\delta t$, which is itself infinitesimal. In particular if we give to $f(x y)$ the values $x$ and $y$ respectively, we obtain $\delta x$ and $\delta y$ from $X f$. It defines then completely the infinitesimal transformations of the group. Roughly speaking, also we may say that the repetition of the infinitesimal transformation an infinite number of times will give a finite transformation of the group; so that they define the finite transformations. This operation is equivalent to an integration, and it is shown that the integration of the simultaneous system

$$
\frac{d x_{1}}{X\left(x_{1}\right)}=\frac{d y_{1}}{X\left(y_{1}\right)}=d t
$$

for the initial conditions that $x_{1}=x$ and $y_{1}=y$ for $t=0$ gives the finite equations of the group. These may be written in the form of series, as follows:

$$
\left\{\begin{array}{l}
x_{1}=x+t X x+\frac{t^{2}}{1.2} X X x+\ldots \\
y_{1}=y+t X y+\frac{t^{2}}{1.2} X X y+\ldots
\end{array}\right.
$$

$X f$ then defines without ambiguity the finite equations of the group also. It defines then the group completely, and is called by Lie the symbol of the group. Its value may be seen at once in answering the question as to what a group becomes when new variables are introduced in place of $x$ and $y$, say $\vec{x}=\lambda_{1}(x y), \bar{y}=\lambda_{2}(x y)$. If $X f$ is the group in $x$ and $y$, the corresponding group in $\bar{x}, \bar{y}$ is

$$
X(\bar{x}) \frac{\partial f}{\partial \bar{x}}+X(\bar{y}) \frac{\partial f}{\partial \bar{y}} .
$$

If we set $X_{i} f=\xi_{i}(x y) p+n_{i}(x y) q$, the equation defining $X f$ takes the form $X f=\sum_{1}^{r} e_{i} X_{i} f$. The $X_{i} f$ are all symbols of infinitesimal transformations and independent, so that from the form of $X f$ the important fact follows that an $r$ parameter group contains exactly $r$ independent infinitesimal transformations. Each of these or in general any $\sum_{1}^{r} e_{i} X_{i} f$, where the $e_{i}$ have particular values generates a one-parameter group which is a subgroup of the $r$-parameter group. 
The problem of determining the subgroups of a given group is a fundamental one in the group theory. One method of doing this is indicated by the relations

$$
\begin{gathered}
\left(X_{i} X_{k}\right)=\sum_{1}^{r} c_{i k s} X_{s} f \\
(i . k=1.2 \ldots r)
\end{gathered}
$$

for if $X_{1} f \ldots X_{q} f$ form a subgroup, then we have these relations holding in particular for $(i, k, s=1.2 \ldots q)$. As a simpler method we have that depending on what Lie calls the adjoined group (adjungirte gruppe). One form of the equations of a group $X_{1} f \ldots X_{r} f$ is

$$
\left\{\begin{array}{c}
x_{1}=x+\sum_{1}^{r} e_{i} \stackrel{r}{X}_{i}(x)+\underset{1}{\sum_{1}^{r}} \sum_{1}^{r} e_{i} e_{k} X_{i} X_{k}(x)+\ldots \\
y_{1}=y+\sum_{1}^{r} e_{i} \stackrel{r}{X}_{i}(y)+\sum_{1}^{r}{\stackrel{\Sigma}{\sum^{k}}}_{1}^{r} e_{i} e_{k} X_{i} X_{k}(y)+\ldots
\end{array}\right.
$$

in which $e_{i} \ldots e_{r}$ are the $r$ parameters of the group. This is called its canonical form, and it has the property that all oneparameter subgroups are of the form $\sum_{1}^{r} e_{i} X_{i} f$; or if in this expression we replace $e_{i}$ by $e_{i}=e^{0}{ }_{i} t$ and allow $t$ alone to vary, we obtain thus all these subgroups. They then are all known. If now on one of these, $\sum_{1}^{r} e_{i} X_{i} f$, we carry out the transformation (1) it will go over into $\sum_{1}^{r} e_{i}^{\prime} X_{i}^{\prime} f$, which will be again a one-parameter subgroup of the group, and we shall have

$$
e_{i}^{\prime}=\sum_{1}^{r} \rho_{i l}\left(a_{1} \ldots a_{r}\right) e_{l}(i=1.2 \ldots r) .
$$

These equations define a group which is linear and homogeneous in $e_{1} \ldots e_{r}$, and determines how the subgroups $\Sigma e_{i} \vec{X}_{i} f$ are interchanged by the transformations of the group. It is called the adjoined group of the given group. Representing by $e_{1} \ldots e_{r}$ the homogeneous co-ordinates in a space of $r-1$ dimensions, it is evident that the points of this space may be taken to represent the one-parameter subgroups, $\Sigma e_{i} X_{i} f$. The adjoined group shows how these are interchanged by the transformations of the group; it shows consequently how the points of the $S_{r_{1}}$ are interchanged. By the adjoined group also any transformation $\Sigma e_{i} X_{i} f$ is regarded not only as an individual 
to be transformed, but also as an operation which it and other individuals of the group undergo. The points of the $S_{r-1}$ represent, then, also transformations of the adjoined group, namely, those which show how the transformations of the original group are interchanged when the transformations of a one-parameter subgroup are carried out on them. Combining these two ways of viewing a point in the $S_{r-1}$, it is shown that an $s$-parameter subgroup of the group is represented by a plane-manifoldness of $s-1$ dimensions in this space, and that for all transformations of the adjoined group which are represented by points of this manifoldness it must be invariant. If in particular this manifoldness remains invariant for all transformations of the adjoined group, the $s$-parameter subgroup is an invariant subgroup of the given group. The determination of all these plane-manifoldnesses of one, two $\ldots r$ dimensions gives then all the one, two...r parameter subgroups of the given group. The problem of finding all these subgroups is thus reduced to that of finding certain invariant manifoldnesses by the adjoined group, a problem susceptible of a complete and comparatively easy solution.

Of the applications treated in the last sections of the book, the question as to when two curves or surfaces are congruent by a Euclidean movement is especially interesting. These movements may be defined as that continuous group of point transformations which transforms any given figure into a congruent figure. The theory of figures congruent by the group is then nothing else than the invariant-theory of the group. In the plane this group has as an invariant the expression for the curvature $\frac{y^{\prime \prime}}{\left(1+y^{\prime 2}\right)^{\frac{3}{2}}}$ or $\frac{1}{r}$. As further invariants come $\frac{d r}{d s}, \frac{d^{2} r}{d s^{2}}$, etc. In space the corresponding group has for invariants $\frac{1}{r}, \frac{d r}{d s}, \ldots \tau, \frac{d \tau}{d s} \ldots$, etc., where $\tau$ is the torsion. The question of the congruency of two curves in the plane, then, depends only on the value of $r$ and $\frac{d r}{d s}$ for the curve; in space further, on the value of the torsion. For corresponding points of the curves the values of these invariants must be the same, or as the correspondence of points of the curves is not known the criteria may be stated, the relations

$$
\frac{d r}{d s}=f(r) \quad \tau=\psi(r),
$$

which determine the manner in which $\frac{d r}{d s}$ and $\tau$ vary with 
$r$ along the curve, must be identically the same for congruent curves. For the exceptional case of constant values of $r$ in the plane we come upon the "minimal lines" $x \pm i y=$ const.

Here it is found that the lines of the two families separately are congruent. In space the minimal curves defined by $\sqrt{d x^{2}+d y^{2}+d z^{2}}=0$ play a similar rôle.

It will be noted that here as in many important applications those invariants known as differential invariants occupy the most prominent position. Under the theory of this class of invariants belongs the entire theory of curvature both of curves and surfaces. Another chapter of the book, although offering no new results, shows also how Cayley's theory of invariants of binary forms can also be brought under this head, being the invariant theory of a linear homogeneous group, and closes by indicating a more general form of this same class of invariants for any group whatever.

We will not take up farther the applications given, but the reader will find extremely interesting and suggestive also the final chapter dealing with the integration theory of Riccati's equation, of systems of linear homogeneous differential equations, and finally that very large class of equations, differential equations with fundamental solutions.

Princeton, June 8, 1895.

J. M. Brooks.

\section{JORDAN'S COURS D'ANALYSE.}

Cours d'Analyse de l'École Polytechnique, par M. C. JoRdan. Deuxieme Edition, entièrement refondue. Tome deuxième, Calcul Intégral. pp. $627+x$ viii.

IN the new edition of M. Jordan's Cours d'Analyse the second volume, like the first, differs greatly in form from that of the original edition. So much is this the case that the present work may be treated as a substantially new contribution to mathematical literature. The character of many of the alterations of previous proofs was foreshadowed by those made in the revised first volume: they are in the direction of increased carefulness and precision of statement. Especially is this the case in the chapter on Definite Integrals. Adopting the definition of $\int_{a}^{b} f(x) d x$ as a limit of sums, the author proceeds to wide generalizations of this definition. The first extension covers the case where $f(x)$ is integrable 